\title{
Vibração ocorrente sobre o corpo inteiro do operador de trator agrícola em ensaio padronizado
}

\author{
Jefferson Sandi ${ }^{1}$, João Vitor Paulo Testa ${ }^{1}$, Murilo Battistuzzi Martins ${ }^{1}$, Diego Augusto \\ Fiorese $^{2}$, Kléber Pereira Lanças ${ }^{1}$ \\ ${ }^{1}$ Universidade Estadual Paulista Júlio de Mesquita Filho, Faculdade de Ciências Agronômicas de Botucatu, Botucatu, São Paulo, \\ Brasil.E-mail: jffsandi@gmail.com, joaovitorpt@ hotmail.com, mbm_martins@hotmail.com, kplancas@fca.unesp.br. \\ ${ }^{2}$ Universidade Federal de Mato Grosso, Câmpus de Sinop, Sinop, Mato Grosso, Brasil. E-mail: dafiorese@ yahoo.com.br
}

Recebido: 10/10/2017; Aceito: 21/03/2018

\section{RESUMO}

Uma das principais variáveis que afetam o rendimento produtivo dos operadores de tratores agrícolas causando sérios problemas de saúde é a vibração gerada durante o funcionamento da máquina. Este trabalho teve como objetivo avaliar a vibração que incide sobre o corpo inteiro do operador através do assento de um trator agrícola mediante ensaio em pista de vibração padronizada. Empregou-se um trator de $92 \mathrm{~kW}$ de potência em quatro condições de lastragem e quatro velocidades de deslocamento. Utilizou-se um acelerômetro de três eixos fixado sobre o assento do trator para avaliação do efeito da vibração no corpo do operador. Foram obtidos os parâmetros aceleração média resultante, valor da dose de vibração, fator de crista e projeção de dose para 8 horas de trabalho. Todas as análises estatísticas foram feitas com o teste de Tukey $(\mathrm{p}<0,05)$. As maiores velocidades geraram as piores condições de trabalho, exceto para a aceleração mínima resultante. A lastragem máxima ocasionou as condições menos prejudiciais quando combinada com as menores velocidades de deslocamento. Os valores de dose de vibração não atingiram o limite de exposição. Na projeção $\mathrm{A}(8)$ todos os tratamentos se encontraram acima do nível de ação ou na região de incerteza.

Palavras-chave: conforto, condições insalubres, ISO 5008, mecanização agrícola, segurança do trabalho.

\section{Vibration over the whole body of the agricultural tractor operator in standardized testing}

\begin{abstract}
One of the main variables affecting the productive performance of agricultural tractor operators that can cause serious health problems is the vibration generated during the operation of this machine. The objective of this study was to evaluate the vibration of the entire body of the operator through the seat of an agricultural tractor in a standardized vibration track test. We used a tractor with $92 \mathrm{~kW}$ of power in the engine in four conditions of ballasting and four speeds of displacement. A three-axis accelerometer was fixed to the tractor seat to assess the effect of vibration on the operator's body. Were acquired the resulting mean acceleration, vibration dose, crest factor and dose projection parameters for 8 hours of work. All statistical analyzes were performed with the Tukey test $(\mathrm{p}<0.05)$. The higher speeds generated the worst working conditions except for the resulting minimum acceleration. Maximum ballasting resulted in less damaging conditions when combined with lower travel speeds. Vibration dose values did not reach the exposure limit. In the projection $\mathrm{A}(8)$ all treatments were above the action level or in the region of uncertainty.
\end{abstract}

Key words: comfort, unhealthy conditions, ISO 5008, agricultural mechanization, work safety. 


\section{Introdução}

Apesar dos ganhos permitidos pela mecanização, muitos fatores afetam as capacidades do operador do trator agrícola durante seu trabalho, sendo a vibração mecânica um deles, causando queda de rendimento e qualidade na operação realizada (KROEMER; GRANDJEAN, 2005). Quatro fatores físicos afetam a resposta humana à vibração: frequência, direção, intensidade e duração; quando um ou mais destes fatores se estabelece acima do nível desejado geram problemas de saúde ao operador (ZEHSAZ et al., 2011).

As vibrações mecânicas originadas tanto no funcionamento da máquina quanto pela rugosidade da superfície de deslocamento se tornam problemáticas quando a frequência de partes do corpo humano (considerando-se que o tronco vibra a uma frequência de 4 a $8 \mathrm{~Hz}$ ), se aproxima à frequência da vibração do trator (1-7 Hz).

Segundo Kroemer e Grandjean (2005), as vibrações afetam a percepção visual, o desempenho psicomotor e muscular e ainda que de forma mais amena, os sistemas circulatório, respiratório e nervoso. Os mesmos autores apontam, em todos os testes de direção em que foram simulados os efeitos psicofisiológicos das vibrações, que a eficiência na direção foi prejudicada por vibrações na faixa de $4 \mathrm{~Hz}$, cujos efeitos negativos crescem com o aumento da aceleração, sendo que os erros de direção aumentam quando o operador está sujeito a acelerações na ordem de $0,5 \mathrm{~m} \mathrm{~s}^{-2}$, tornando impossível guiar com segurança quando as acelerações atingem $2,5 \mathrm{~m} \mathrm{~s}^{-2}$.

Grande parte destes problemas podem ser evitados por meio de levantamentos de dados das condições de trabalho e pela adoção de valores limites para os níveis de exposição. A redução da vibração possibilita melhor aproveitamento da jornada de trabalho, maior conforte e consequentemente reduz o cansaço do operador (TEWARI; DEWANGAN, 2009). Mehta et al. (2000), verificaram que de acordo com a ISO 2631-1, os níveis de vibrações em tratores agrícolas nos eixos longitudinal, transversal e vertical na interface assentooperador, em diferentes condições de operação, expõem os operadores de tratores agrícolas a diversas desordens na coluna vertebral.

Na ISO 2631 (1978), são definidos três limites de exposição do corpo humano às vibrações buscando estabelecer os níveis de conforto, eficiência de trabalho e risco a saúde, sendo eles: nível de conforto reduzido (preservação do conforto), nível de eficiência reduzida/fadiga (preservação da eficiência de trabalho) e limite de exposição (preservação da saúde e/ou segurança). Estes limites são estabelecidos de acordo com os níveis de frequência vibratória, acelerações, tempo de exposição e direção da vibração em relação ao tronco. A norma em questão também pede que as medições sejam feitas o mais próximo possível do ponto ou área na qual a vibração é transmitida ao corpo para se conhecer o seu nível real de ação. Segundo Nietiedt et al. (2012), utiliza-se o Ponto de Referência do Assento (SIP) definido pela Norma ISO 5353 (1999) quando deseja-se obter os valores de vibração que incidem sobre o corpo inteiro do operador durante a operação de uma máquina agrícola.

Segundo Cunha et al. (2012), os problemas com vibrações sobre o operador podem ser atenuados com a redução da intensidade da mesma na fonte combinada à diminuição do tempo de exposição, sendo necessário para isso, conhecer a realidade das máquinas atuais no que se refere à vibração para planejar a melhor forma de mitigar este problema. Porém, ainda em alguns países, devido às razões econômicas, um trator sem suspensão na cabine e assento tem maior demanda, fazendo com que os fabricantes decidam por disponibilizar apenas o isolamento da cabine contra poeira e água (ZEHSAZ et al., 2011).

Para Silva et al. (2011), os assentos contribuíram de forma significativa para a mudança comportamental através do fornecimento de melhor estabilidade do corpo, porém ocasionou problemas ergonômicos para a disposição dos comandos.

Santos Filho et al. (2003), observando os níveis de vibração vertical no assento de um trator agrícola e na base do assento gerados durante a operação de gradagem empregando diferentes velocidades de trabalho, concluíram que os níveis de aceleração encontrados na parte superior do assento foram inferiores aos níveis da base para todas as velocidades, tendo a marcha mais veloz apresentado maiores níveis de vibração. A faixa de frequência que resultou nos maiores picos de vibração vertical ficou situada entre 2 e $4 \mathrm{~Hz}$; os valores de aceleração ponderada global encontraram-se bem acima dos limites definidos pela ISO 2631-1 para 8 horas de trabalho diário e o aumento da velocidade de deslocamento do conjunto ocasionou o aumento dos níveis de vibração.

No Brasil, as Normas Regulamentadoras - NR relativas à segurança e medicina do trabalho abordam aspectos relacionados à insalubridade das atividades trabalhistas. A Norma Regulamentadora $n^{\text {o }} \quad 15$ estabelece os limites de tolerância para cada atividade de acordo com a concentração ou intensidade de exposição do trabalhador ao agente nocivo. Porém, esta norma não se refere a limites de exposição à vibração, o que leva a adoção das normas internacionais para realização dos ensaios, dentre elas a ISO 2631 e a diretiva 2002/44/CE da Comunidade Europeia que estipulam níveis de ação e limites de exposição humana às vibrações do corpo inteiro (SANTOS et al., 2014).

Na Legislação Nacional, a Norma Regulamentadora 31 (2005) define as responsabilidades, ações e direitos dos trabalhadores, empregadores e órgãos fiscalizadores 
em relação às atividades trabalhistas desempenhadas no meio rural. Esta norma tem como objetivo estabelecer os preceitos a serem observados e seguidos na organização e no ambiente de trabalho para tornar compatível o planejamento e o desenvolvimento das atividades agrícolas com a segurança e saúde no ambiente de trabalho.

Assim, este trabalho teve como objetivo estudar a vibração mecânica incidente sobre o operador de um trator agrícola $4 \times 2$ TDA com cabine ensaiado em pista de vibração com diferentes lastros, velocidades e pressões de inflação dos pneus.

\section{Material e Métodos}

O ensaio foi realizado na Faculdade de Ciências Agronômicas da Universidade Estadual Paulista "Júlio de Mesquita Filho", Campus de Botucatu - SP. As coordenadas geográficas da área experimental onde a pista foi instalada são: $22^{\circ} 51^{\prime} \mathrm{S}$ e $48^{\circ} 25^{\prime} \mathrm{W}$; a altitude do local é de 770 metros.

A coleta de dados foi realizada sobre pista para ensaio de vibração em tratores agrícolas construída em madeira segundo a Norma ISO 5008 de 2002 (Figura 1). A disposição dos degraus de madeira de acordo com sua elevação ao longo dos dois trechos de deslocamento obedeceu à ordem estabelecida pela norma.

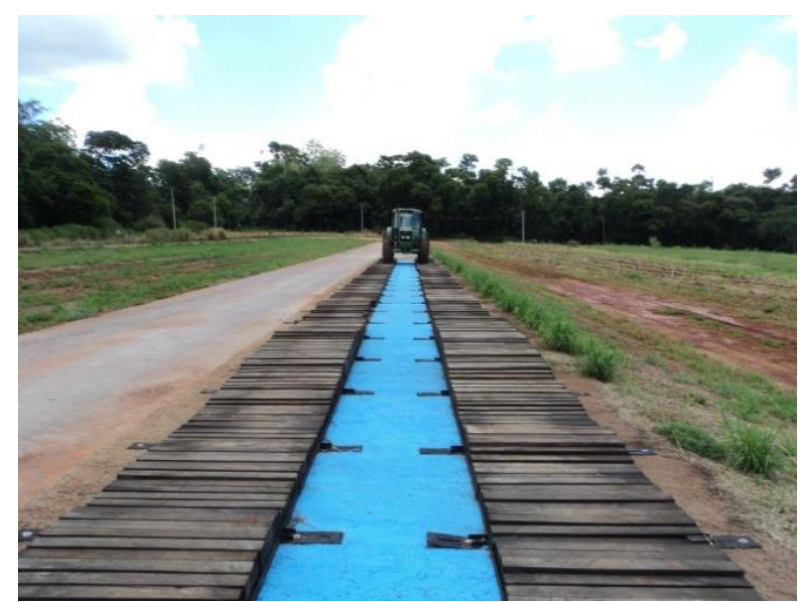

Figura 1. Pista de vibração utilizada para ensaio. FCA Botucatu (2015).

Foi utilizado um trator agrícola 4x2 TDA com 92 kW de potência no motor, utilizando um conjunto de pneus diagonais TM 95 (dianteiros 14.9-24 e traseiros 23.1-30). O assento presente no trator pertence à família de assentos FA 418/631, fabricado pela Grammer AG do Brasil. Para aquisição dos dados de desempenho operacional empregou-se um acelerômetro de três eixos (X, Y e Z) - HVM100 Larson Davis e software Blaze 6.1.1 para transferência de dados. Para avaliação da vibração foi utilizado um acelerômetro de três eixos (x, y, z) HVM100 em conjunto com acelerômetro SEN027
- Seat Pad, ambos fabricados pela Larson Davis. O aparelho foi calibrado antes dos ensaios. $\mathrm{O}$ equipamento HMV100 foi configurado para operar com o modo de corpo inteiro, média lenta, acelerômetro ICP, com referência de exposição de $2,8 \mathrm{~m} \mathrm{~s}^{2}$, sem integração, ponderação de frequência $\mathrm{Wm}$ Prédios, fatores multiplicativos de 1,4 (para os eixos x e y) e 1 (eixo z), sem ganho e saída com AC ponderado.

O sistema de medição para corpo inteiro (whole body) foi fixado sobre o assento do operador de acordo com a Norma de Higiene Ocupacional 09 (FUNDACENTRO, 2013) com o acelerômetro tri-axial (disco flexível, com um acelerômetro central) para mensurar a vibração aplicada a todo o corpo humano, de acordo com os três eixos. As coordenadas cartesianas que foram obedecidas na fixação do acelerômetro ao assento foram: eixo $\mathrm{x}$ - frente/atrás; eixo $\mathrm{y}$ direita/esquerda; eixo z - para cima/para baixo. Para evitar a movimentação do disco, este foi fixado por meio de fita adesiva ao assento. Em seguida, fez-se o ajuste no aparelho coletor dos dados dos parâmetros referentes à vibração no corpo todo, a medição nos três eixos cartesianos e o intervalo de aquisição dos dados. $\mathrm{O}$ acionamento da aquisição dos dados foi realizado momentos antes da passagem pela pista, sendo desligado logo após o término da passagem sobre a mesma.

Os parâmetros avaliados durante o deslocamento do trator sobre a pista de vibração foram: valores de aceleração média resultante, valor de fator de crista, valor da dose de vibração e a projeção de dose para 8 horas de trabalho. A aceleração média resultante é corresponde à raiz quadrada da soma dos quadrados das acelerações médias, medidas segundo os três eixos ortogonais " $x "$, " $y$ " e " $z$ ", enquanto as acelerações máxima e mínima expressam os valores máximos e mínimos da raiz quadrada da soma dos quadrados das acelerações obtidas segundo os três eixos ortogonais "x", "y" e "z".

$\mathrm{O}$ fator de crista permite determinar a homogeneidade da vibração ao longo de um período no cenário em estudo, através da ocorrência de pico destacados, que podem ser resultantes de fenômenos repetitivos a intervalos regulares. A Norma de Higiene Ocupacional 09, (FUNDACENTRO, 2013) estabelece que valores de fator de crista acima de 9,0 devem ser avaliados cuidadosamente. $\mathrm{Na}$ Tabela 1 são apresentados os critérios para tomada de decisão baseados na aceleração resultante de exposição normalizada (aren), e valor de dose de vibração resultante (VDVR), além do conjunto mínimo de medidas preventivas e corretivas que devem ser adotados quando constadas as condições com níveis de vibrações prejudiciais. 
Tabela 1. Critério de julgamento e tomada de decisão

\begin{tabular}{cccc}
\hline aren $\left(\mathrm{m} \mathrm{s}^{-2}\right)$ & VDVR $\left(\mathrm{m} \mathrm{s}^{-1,75}\right)$ & Consideração Técnica & Atuação Recomendada \\
\hline 0 a 0,5 & 0 a 9,1 & Aceitável & $\begin{array}{c}\text { No mínimo manutenção da condição } \\
\text { existente }\end{array}$ \\
\hline$>0,5$ a $<0,9$ & $>9,1$ a $<16,4$ & Acima do nível de ação & No mínimo adoção de medidas preventivas \\
\hline 0,9 a 1,1 & 16,4 a 21 & Região de incerteza & $\begin{array}{c}\text { Adoção de medidas preventivas e corretivas } \\
\text { visando a redução da exposição diária }\end{array}$ \\
\hline Acima de 1,1 & Acima de 21 & Acima do limite de exposição & Adoção imediata de medidas corretivas \\
\hline
\end{tabular}

Fonte: Norma de Higiene Ocupacional 09 (FUNDACENTRO, 2013).

O valor da dose de vibração (VDV) é o valor obtido a partir do método de dose de vibração à quarta potência determinada na direção " $\mathrm{i}$ ", onde "i" corresponde aos

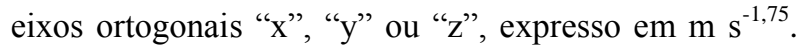
Nas normas internacionais adota-se o valor dose de vibração como sendo o maior valor de dose de vibração obtido entre os três eixos ortogonais enquanto a Norma de Higiene Ocupacional 09 (FUNDACENTRO, 2013) obtém este valor através do conceito de valor dose resultante (VDVR).

A projeção $\mathrm{A}(8)$, é um método de avaliação que gera um valor médio de vibração ajustado para representar a dose de uma jornada de trabalho de oito horas, pois no banco do operador os valores se mantêm de forma relativamente estável durante este período (SCARLETT et al., 2007).

O trator foi ensaiado em pista de vibração com quatro condições de lastragem: completamente lastrado através da combinação de uso de lastragem líquida e sólida (L1), empregando somente lastro sólido (L2); somente lastro líquido (L3); completamente sem lastros (L4), resultando nas massas de: 70; 54; 54 e $48 \mathrm{kN}$ respectivamente. Foram quatro velocidades de deslocamento: 1,19 $\mathrm{m} \mathrm{s}^{-1}$ (V1); 1,47 $\mathrm{m} \mathrm{s}^{-1}$ (V2); 1,75 m $\mathrm{s}^{-1}$ (V3) e 2,08 $\mathrm{m} \mathrm{s}^{-1}$ (V4), com a pressão de inflação de 137,85 e $165,47 \mathrm{kPa}$ nos rodados traseiros e dianteiros respectivamente. Em todas as situações adotou-se a mesma distribuição de $35 \%$ do peso no eixo dianteiro e $65 \%$ no eixo traseiro. O delineamento experimental foi completamente casualizado, em esquema fatorial, com cinco repetições. Todas as análises estatísticas foram feitas com Tukey a $5 \%$ de probabilidade $(\mathrm{p}<0,05)$ com auxílio do software Minitab ${ }^{\circledR}$ 16.2.4.4.

\section{Resultados e Discussão}

Pode-se observar na Tabela 2, que os menores valores de aceleração média resultante foram obtidos na combinação entre a menor velocidade de deslocamento (V1) e a lastragem L1, e na lastragem L2 em combinação com a velocidade V2. Os maiores valores de aceleração média resultante ocorreram na combinação das lastragens L3 e L4 com a velocidade V4.
Tabela 2. Valores de soma de aceleração média resultante (m $\mathrm{s}^{2}$ ) para as diferentes lastragens e velocidades de deslocamento

\begin{tabular}{cllll}
\hline \multirow{2}{*}{ Lastragem } & \multicolumn{4}{c}{ Velocidade } \\
\cline { 2 - 5 } & V1 & V2 & V3 & V4 \\
\hline L1 & $2,81 \mathrm{Bd}$ & $3,21 \mathrm{Cc}$ & $4,16 \mathrm{Bb}$ & $5,49 \mathrm{Ba}$ \\
L2 & $2,86 \mathrm{Bd}$ & $3,49 \mathrm{Bc}$ & $4,34 \mathrm{Bb}$ & $5,57 \mathrm{Ba}$ \\
L3 & $3,10 \mathrm{Ad}$ & $3,52 \mathrm{Bc}$ & $4,67 \mathrm{Bb}$ & $5,89 \mathrm{Aa}$ \\
L4 & $3,19 \mathrm{Ad}$ & $3,90 \mathrm{Ac}$ & $4,99 \mathrm{Ab}$ & $6,06 \mathrm{Aa}$ \\
\hline F de Lastragem & & $140,34^{* *}$ \\
F de Velocidade & & $2832,04 * *$ \\
F de Lastragem * Velocidade & \multicolumn{3}{c}{$4,66^{* *}$} \\
\hline CV(\%) & \multicolumn{3}{c}{2,43} \\
\hline
\end{tabular}

Médias seguidas de mesma letra maiúscula não diferem entre si na coluna e mesma letra minúscula não diferem entre si na linha pelo teste Tukey $(p \leq 0,05)$. ns: não significativo pelo teste $\mathrm{F}$ ao nível de $5 \%$ de probabilidade. * Significativo pelo teste $\mathrm{F}$ ao nível 5\% de probabilidade. ** Significativo pelo teste $\mathrm{F}$ ao nível de $1 \%$ de probabilidade. L1: lastro máximo; L2: somente lastro sólido; L3: somente lastro líquido; L4: sem lastros adicionais. V1: 1,19 $\mathrm{m} \mathrm{s}^{-1}$; V2: $1,47 \mathrm{~m} \mathrm{~s}^{-1}$; V3: $1,75 \mathrm{~m} \mathrm{~s}^{-1}$; V4: $2,08 \mathrm{~m} \mathrm{~s}^{-1}$.

Segundo Kroemer e Grandjean (2005), em todos os ensaios em que foram simulados os efeitos psicofisiológicos das vibrações, a eficiência na direção foi prejudicada por vibrações na faixa de $4 \mathrm{~Hz}$, onde os efeitos negativos crescem com o aumento da aceleração, sendo que os erros de direção aumentaram quando o operador está sujeito a acelerações na ordem de $0,5 \mathrm{~m} \mathrm{~s}^{-}$ ${ }^{2}$, tornando impossível dirigir com segurança quando as acelerações atingem $2,5 \quad \mathrm{~m} \mathrm{~s}^{-2}$. Os resultados encontrados evidenciaram que, em todas as condições, os valores de aceleração se encontram acima do nível adequado para permitir que o operador possa operar o trator com segurança.

$\mathrm{O}$ maior peso e a menor velocidade podem ser fatores que proporcionem menor energia inercial e maior estabilidade ao trator durante seu deslocamento, permitindo que os pneus possam se adaptar melhor ao terreno através de sua deformação vertical e horizontal para absorver de forma mais eficiente as imperfeições existentes, diminuindo a energia e intensidade das vibrações que chegam até o operação. Por outro lado, a maior velocidade foi determinante em todas as pressões ensaiadas para obtenção dos maiores valores de acelerações médias devido à maior energia inercial e movimentos mais rápidos ocasionados nesta situação. 
Pinho et al. (2014) indicaram que os menores valores de vibração, quando se utiliza o lastro metálico total ocorreram devido à maior tendência inercial em função da aceleração longitudinal inversamente proporcional à massa do trator e diretamente à força motora dele; quando se utiliza a mesma relação massa/potência, com o lastro liquido, o movimento oscilatório da água no interior do pneu, na direção de deslocamento, produziu um aumento da magnitude das acelerações. Os altos valores de aceleração média resultante obtidos neste ensaio em pista de vibração de $35 \mathrm{~m}$ se devem às características da pista, que busca simular uma condição de grande irregularidade no terreno de trabalho, como por exemplo as encontradas em um solo recém trabalhado com arados e subsoladores ou quando existem obstáculos em meio ao campo, como canais de assoreamento, canteiros, camaleões, pedras, galhos e tocos. Kumar et al. (2001) obtiveram em seus ensaios, valores de aceleração média entre 1,58 e $4,57 \mathrm{~m} \mathrm{~s}^{-2}$ em pista de vibração padronizada, estradas pavimentadas e terreno coberto por palhada. Estes autores apontam que ensaios de vibração realizados durante as operações agrícolas apresentam menores valores posto que os implementos atuam como atenuadores da vibração. Os maiores valores de fator de crista foram observados na velocidade V4 e no tratamento com maior lastragem (L1) juntamente com a maior velocidade V4 (Tabela 3).

É possível notar que o valor estabelecido pela Norma de Higiene Ocupacional 09 (FUNDACENTRO, 2013) é ultrapassado na condição L4 na combinação com a velocidade V3 e em todas as condições de lastragens na Velocidade V4. Nestes tratamentos ocorreram picos de acelerações que tornaram o valor de dose da vibração um parâmetro muito importante para determinar a insalubridade da operação. Estes picos de aceleração indicam a ocorrência de vibrações que podem ser prejudiciais ao operador. Segundo Koley et al. (2010), os próprios operadores de tratores agrícolas devem tomar precauções e cuidados com sua coluna vertebral, principalmente devido às vibrações de baixa frequência durante longos períodos que podem resultar em problemas degenerativos neste órgão.

Os problemas causados pelas vibrações são mais graves em tratores convencionais (de menor aporte tecnológico), pois segundo Cuong et al. (2013), estas máquinas apresentam quase que exclusivamente os pneus como componente elástico entre a estrada e o trator, sendo que os mesmos, muitas vezes são incapazes de proporcionar as características de suspensão adequados para absorver as vibrações ocasionadas durante o deslocamento.

Na Tabela 4 são apresentados os valores da dose de vibração obtidos durante o ensaio em pista de vibração de 35 metros.
Tabela 3. Valores de fator de crista $(\mathrm{dB})$ para diferentes lastragens e velocidades de deslocamento.

\begin{tabular}{ccccc}
\hline \multirow{2}{*}{ Lastragem } & \multicolumn{4}{c}{ Velocidade } \\
\cline { 2 - 5 } & $\mathrm{V} 1$ & $\mathrm{~V} 2$ & $\mathrm{~V} 3$ & $\mathrm{~V} 4$ \\
\hline $\mathrm{L} 1$ & $7,9 \mathrm{Ab}$ & $8,2 \mathrm{Ab}$ & $7,6 \mathrm{Bb}$ & $10,1 \mathrm{Aa}$ \\
$\mathrm{L} 2$ & $7,9 \mathrm{Ab}$ & $7,6 \mathrm{Ab}$ & $8,0 \mathrm{ABb}$ & $9,7 \mathrm{Aa}$ \\
$\mathrm{L} 3$ & $7,6 \mathrm{Ab}$ & $8,2 \mathrm{Aab}$ & $8,4 \mathrm{ABab}$ & $9,3 \mathrm{Aa}$ \\
$\mathrm{L} 4$ & $7,1 \mathrm{Ab}$ & $8,7 \mathrm{Aa}$ & $9,3 \mathrm{Aa}$ & $9,7 \mathrm{Aa}$ \\
\hline F de Lastragem & & $2,14 \mathrm{~ns}$ \\
F de Velocidade & & $37,57^{* *}$ \\
Lastragem * Velocidade & & $3,17^{*}$ \\
\hline CV(\%) & & 7,57 \\
\hline
\end{tabular}

Médias seguidas de mesma letra maiúscula não diferem entre si na coluna e mesma letra minúscula não diferem entre si na linha pelo teste Tukey $(\mathrm{p} \leq 0,05)$. ns: não significativo pelo teste $\mathrm{F}$ ao nível de $5 \%$ de probabilidade. * Significativo pelo teste $\mathrm{F}$ ao nível $5 \%$ de probabilidade. ** Significativo pelo teste $\mathrm{F}$ ao nível de $1 \%$ de probabilidade. L1: lastro máximo; L2: somente lastro sólido; L3: somente lastro líquido; L4: sem lastros adicionais. V1: $1,19 \mathrm{~m} \mathrm{~s}^{-1} ; \mathrm{V} 2$ : $1,47 \mathrm{~m} \mathrm{~s}^{-1} ; \mathrm{V} 3: 1,75 \mathrm{~m} \mathrm{~s}^{-1} ; \mathrm{V} 4: 2,08 \mathrm{~m} \mathrm{~s}^{-1}$.

Tabela 4. Valores da dose de vibração $\left(\mathrm{m} \mathrm{s}^{-1,75}\right)$ de lastragens e velocidades de deslocamento

\begin{tabular}{ccccc}
\hline \multirow{2}{*}{ Lastragem } & \multicolumn{4}{c}{ Velocidade } \\
\cline { 2 - 5 } & $\mathrm{V} 1$ & $\mathrm{~V} 2$ & $\mathrm{~V} 3$ & $\mathrm{~V} 4$ \\
\hline $\mathrm{L} 1$ & $7,79 \mathrm{Bd}$ & $8,83 \mathrm{Cc}$ & $10,66 \mathrm{Cb}$ & $13,92 \mathrm{Ca}$ \\
$\mathrm{L} 2$ & $8,28 \mathrm{Ad}$ & $9,27 \mathrm{BCc}$ & $10,98 \mathrm{Cb}$ & $14,12 \mathrm{Ca}$ \\
$\mathrm{L} 3$ & $8,35 \mathrm{Ad}$ & $9,57 \mathrm{Bc}$ & $11,88 \mathrm{Bb}$ & $14,66 \mathrm{Ba}$ \\
L4 & $8,65 \mathrm{Ad}$ & $10,44 \mathrm{Ac}$ & $12,72 \mathrm{Ab}$ & $15,24 \mathrm{Aa}$ \\
\hline F de Lastragem & \multicolumn{3}{c}{$19,73^{* *}$} \\
F de Velocidade & & $365,22^{* *}$ \\
Lastragem * Velocidade & & $8,89^{* *}$ \\
\hline CV $(\%)$ & & & 1,83 \\
\hline
\end{tabular}

Médias seguidas de mesma letra maiúscula não diferem entre si na coluna e mesma letra minúscula não diferem entre si na linha pelo teste Tukey $(\mathrm{p} \leq 0,05)$. ns: não significativo pelo teste $\mathrm{F}$ ao nível de $5 \%$ de probabilidade. * Significativo pelo teste $\mathrm{F}$ ao nível $5 \%$ de probabilidade. ** Significativo pelo teste $\mathrm{F}$ ao nível de $1 \%$ de probabilidade. L1: lastro máximo; L2: somente lastro sólido; L3: somente lastro líquido; L4: sem lastros adicionais. V1: 1,19 $\mathrm{m} \mathrm{s}^{-1}$; V2: $1,47 \mathrm{~m} \mathrm{~s}^{-1} ; \mathrm{V} 3: 1,75 \mathrm{~m} \mathrm{~s}^{-1} ; \mathrm{V} 4: 2,08 \mathrm{~m} \mathrm{~s}^{-1}$.

Novamente o melhor cenário ocorreu na interação entre a lastragem máxima (L1) e a menor velocidade (V1), enquanto a ausência de lastros (L4) com a velocidade V4 resultou no maior valor de dose de vibração. O VDV representa a exposição cumulativa da exposição à vibração em um dia de trabalho, sendo mais indicado para avaliação do efeito da vibração sobre o corpo humano em jornadas maiores que 8 horas por representar de forma mais adequada os solavancos e choques que ocorrem durante o trabalho (SCARLETT et al., 2007). Dessa forma é importante ressaltar que durante o ensaio foram encontrados valores de fator de crista maiores do que nove $(\mathrm{FC}>9,0)$ para muitos tratamentos.

Os tratamentos da velocidade V1 e a lastragem máxima (L1) em combinação com a velocidade V2 apresentaram valores abaixo do nível de ação $\left(9,1 \mathrm{~m} \mathrm{~s}^{-}\right.$ 
${ }^{1,75}$ ), não sendo necessárias medidas para atenuar os valores de vibração incidentes sobre o corpo inteiro do operador; os demais tratamentos, acima do nível de ação, sem contudo atingir a região de incerteza (faixa entre os valores acima do nível de ação e o limite de exposição de $21 \mathrm{~m} \mathrm{~s}^{-1,75}$ ), o que implica na adoção de medidas preventivas tais como: redução da velocidade de trabalho, verificação da correta regulagem e posição do assento para operador, buscar , sempre que possível superfícies mais regulares.

As situações de exposição ocupacional superiores ao nível de ação implicam na adoção obrigatória de medidas de caráter preventivo, enquanto os cenários de exposição ocupacional superiores ao limite de exposição implicam na obrigatória adoção de medidas de caráter corretivo. Os valores encontrados se deram pela forma construtiva da pista de vibração de $35 \mathrm{~m}$, que buscou simular uma superfície acidentada, bem próxima à que os tratores normalmente encontram no campo.

Em experimento realizado por Ribas et al. (2014), utilizando tratores, foram encontrados valores de VDVs superiores a $8,5 \mathrm{~m} \mathrm{~s}^{-1,75}$ no assento e no seu suporte. Metha et al. (2000), encontraram valores de dose de vibração para oito horas de trabalho que ultrapassaram o valor de $15 \mathrm{~m} \mathrm{~s}^{-1.75}$ durante o deslocamento do trator nas velocidades de 1,$64 ; 1,79$ e $0,51 \mathrm{~m} \mathrm{~s}^{-1}$ em estrada de asfalto, estrada de fazenda e de campo, respectivamente, e também durante operação de aração e gradagem.

Na Tabela 5 são apresentados os dados de valores de projeção de dose para oito horas de trabalho $\mathrm{A}(8)$ em que todos os tratamentos se encontram acima do nível de ação ou na faixa de incerteza.

Os tratamentos geraram valores menores que $1,1 \mathrm{~m}$ s-2, o que implica na necessidade de intervenções com caráter preventivo para as lesões e problemas de saúde aos operadores, como adoção de menores velocidades de operação, treinamento e conscientização dos operadores sobre a importância da adoção de boas práticas de trabalho e da seleção correta das regulagens e condições de trabalho. Os resultados obtidos demonstraram que a pista de vibração padronizada de 35 metros pode ser empregada como uma forma de avaliar os tratores para jornadas de trabalho de $8 \mathrm{hs}$.

Santos Filho et al. (2003), em experimento utilizando diferentes marchas para deslocamento do trator durante operação de gradagem sobre solo arado, obtiveram valores de aceleração ponderada global bem acima dos limites determinados para uma exposição de 4 horas de trabalho, de acordo com a ISO 2631 (1997). O emprego da terceira marcha resultou em uma aceleração ponderada global de $2,64 \mathrm{~m} \mathrm{~s}^{-2}$; para a segunda marcha foi $2,10 \mathrm{~m} \mathrm{~s}^{-2}$ e para a primeira marcha $1,73 \mathrm{~m} \mathrm{~s}^{-2}$. Cunha et al. (2009) obtiveram resultados onde o limite para oito horas diárias de trabalho foi excedido, sendo que as piores situações ocorreram com as maiores acelerações ocorrendo no sentido de deslocamento do trator a medida que a rotação do motor foi aumentada. Para Scarlett et al. (2007), a maioria das empresas fabricantes de tratores agrícolas incorpora e utiliza sistemas e mecanismos para reduzir o efeito das vibrações sobre o operador.

Tabela 5. Valores de projeção de dose para jornada de oito horas de trabalho $\mathrm{A}(8)(\mathrm{m} \mathrm{s}-2)$ para diferentes lastragens e velocidades de deslocamento.

\begin{tabular}{|c|c|c|c|c|}
\hline \multirow{2}{*}{ Lastragem } & \multicolumn{4}{|c|}{ Velocidade } \\
\hline & V1 & $\mathrm{V} 2$ & V3 & V4 \\
\hline L1 & $0,7 \mathrm{Cc}$ & $0,7 \mathrm{Cb}$ & $0,8 \mathrm{Cb}$ & $1,0 \mathrm{Ba}$ \\
\hline L2 & $0,7 \mathrm{Bc}$ & $0,8 \mathrm{Bb}$ & $0,9 \mathrm{Bb}$ & 1,0 Aba \\
\hline L3 & $0,7 \mathrm{Bc}$ & $0,8 \mathrm{Bb}$ & $1,0 \mathrm{Aa}$ & $1,0 \mathrm{Aba}$ \\
\hline $\mathrm{L} 4$ & $0,8 \mathrm{Ac}$ & $0,9 \mathrm{Ab}$ & $1,0 \mathrm{Aa}$ & $1,0 \mathrm{Aa}$ \\
\hline \multicolumn{3}{|l|}{ F de Lastragem } & \multicolumn{2}{|c|}{$112,85 * *$} \\
\hline \multicolumn{3}{|l|}{ F de Velocidade } & \multicolumn{2}{|c|}{$578,57 * *$} \\
\hline \multicolumn{3}{|c|}{ F de Lastragem $*$ Velocidade } & \multicolumn{2}{|c|}{$11,61 * *$} \\
\hline \multicolumn{3}{|l|}{$\mathrm{CV}(\%)$} & \multicolumn{2}{|c|}{2,32} \\
\hline \multicolumn{5}{|c|}{$\begin{array}{l}\text { Médias seguidas de mesma letra maiúscula não diferem entre si na } \\
\text { coluna e mesma letra minúscula não diferem entre si na linha pelo } \\
\text { teste Tukey }(\mathrm{p} \leq 0,05) \text {. ns: não significativo pelo teste } \mathrm{F} \text { ao nível de } 5 \% \\
\text { de probabilidade. * Significativo pelo teste } \mathrm{F} \text { ao nível } 5 \% \text { de } \\
\text { probabilidade. ** Significativo pelo teste } \mathrm{F} \text { ao nível de } 1 \% \text { de } \\
\text { probabilidade. L1: lastro máximo; L2: somente lastro sólido; L3: } \\
\text { somente lastro líquido; L4: sem lastros adicionais. V1: } 1,19 \mathrm{~m} \mathrm{~s}^{-1} \text {; V2: } \\
1,47 \mathrm{~m} \mathrm{~s}^{-1} ; \mathrm{V} 3: 1,75 \mathrm{~m} \mathrm{~s}^{-1} ; \mathrm{V} 4: 2,08 \mathrm{~m} \mathrm{~s}^{-1} \text {. }\end{array}$} \\
\hline
\end{tabular}

Marsili et al. (2002), demostraram que a adoção de sistemas de suspensão pode permitir um incremento superior a $50 \%$ no tempo de exposição do operador ao trabalho. Para Metha et al. (2000), cada pesquisador possui uma visão diferente sobre qual o melhor procedimento para predizer o desconforto ocasionado pela vibração em tratores agrícolas, porém, as Normas ISO 2631-1 e BS 6841 apresentam critérios para predizer a vibração incidente sobre o operador.

Os resultados obtidos neste trabalho estão de acordo com os encontrados por Cunha et al. (2012), cujos valores de vibração medidos no trator mais novo empregado no ensaio não atingiram os limites indicados pelas normas. Segundo este mesmo autor, tal fato indica que os avanços tecnológicos, principalmente nos sistemas de suspensão e assentos dos tratores têm proporcionado maior conforto ao operador garantindo a redução da exposição do operador a níveis de vibração prejudiciais à saúde e segurança.

\section{Conclusões}

As maiores velocidades geraram as piores condições de trabalho em todos os parâmetros avaliados, exceto para a aceleração mínima resultante.

A lastragem máxima do trator ocasionou as condições menos prejudiciais ao operador quando combinada com as menores velocidades de deslocamento do trator. 
Ocorreram valores de fator de crista maior que nove, indicando a ocorrência de picos de vibração e a necessidade de avaliação pelo valor de dose de vibração. Os valores de dose de vibração não atingiram o limite de exposição.

$\mathrm{Na}$ projeção de dose para oito horas de trabalho, todos os tratamentos alcançaram nível de ação acima do desejado ou na região de incerteza.

\section{Referências Bibliográficas}

MTE. MINISTÉRIO DO TRABALHO E EMPREGO. Norma regulamentadora de segurança e saúde no trabalho na agricultura, pecuária, silvicultura, exploração florestal e aquicultura - NR 31. 2005. Disponível em: < http://trabalho.gov.br/images/Documentos/SST/NR/NR31.pdf >. Acesso em: 02 out. 2017.

CUONG. D. M.; ZHU, S.; ZHU, Y. Effects of tyre inflation pressure and forward speed on vibration of an unsuspended tractor. Journal of Terramechanics. Silsoe. v. 50, p. 185198. 2013.

CUNHA, J. P. A. R.; DUARTE, M. A. V.; SOUZA, C. M. A Vibração e ruído emitidos por dois tratores agrícolas. IDESIA. Tarapacá, Chile, v. 30, n. 1, p. 25-34. 2012.

CUNHA, J. P. A. R.; DUARTE, M. A. V.; RODRIGUES. J. C. Avaliação dos níveis de vibração e ruído emitidos por um trator agrícola em preparo de solo. Pesquisa Agropecuária Tropical, Goiânia-GO, v. 39, n. 4, p. 348-355, 2009.

FUNDACENTRO. Norma de Higiene Ocupacional NHO 09: avaliação da exposição ocupacional a vibrações de corpo inteiro: procedimento técnico. São Paulo-SP: Fundacentro, 2013. $63 \mathrm{p}$.

ISO. INTERNATIONAL ORGANIZATION FOR STANDARDIZATION. ISO 5008: Agricultural wheeled tractors and field machinery - measurement of whole-body vibration of the operator; Geneva, Switzerland, 2002. 187 p.

ISO. INTERNATIONAL ORGANIZATION FOR STANDARDIZATION. ISO 2631-1: Mechanical vibration and shock - evaluation of human exposure to whole body vibration - Part I: general requirements. Geneva, Switzerland: International Standard, 1997. 23 p.

ISO. INTERNATIONAL ORGANIZATION FOR STANDARDIZATION. ISO 2631: "Guide for evaluation of human exposure to whole-body vibration". Geneva, Switzerland: International Standard, 1978. 18 p.

ISO. INTERNATIONAL ORGANIZATION FOR STANDARDIZATION. ISO 5353: "Earth-moving machinery, tractors and machinery for agriculture and forestry - Seat index point". Geneva, Switzerland: International Standard. 1999. 5 p.

KOLEY, S.; SHARMA, L.; KAUR, S. Effects of occupational exposure to whole-body vibration in tractor drivers with low back pain in Punjab. The Anthropologist, Haryana, India, v. 12, n. 3, p. 183-187. 2010.
KROEMER, K. H. E.; GRANDJEAN, E. Manual de Ergonomia: adaptando o trabalho ao homem. 5. ed. Porto Alegre-RS: Artmed Editora. 2005. 327 p.

KUMAR, A. MAHAJAN, P; MOHAN, D.; VARGHESE, M. Tractor vibration severity a driver healt: a study from rural India. Journal of Agricultural Engineering Research, Pavia, ITA, v. 80, n. 4, p. 313-328, 2001.

MARSILI, A. RAGNI, L.; SANTORO, G.; SERVADIO, P.; VASSALINI, G. Innovative systems to reduce vibrations on agricultural tractors: comparative analysis of acceleration transmitted through the driving seat. Biosystems Engineering. UK. v. 81, p. 35-47, 2002.

MEHTA, C. R.; SHYAM, M.; VERMA, R. N., Ride vibration on tractor-implement system. Applied Ergonomics. Silsoe, n. 31 , p. $323-328,2000$

NIETIEDT, G. H.; RIBAS, R. L. SCHLOSSER, J. F.; FRANTZ, U. G.; CASALI, A. L.; UHRY, D. Distribuição dos comandos de operação em tratores agrícolas nacionais com até $55 \mathrm{~kW}$ de potência. Revista Brasileira de Engenharia Agrícola e Ambiental, Campina Grande-PB, v. 16, n. 6, p. 690-695, 2012.

PINHO, M. S.; SCHLOSSER, J. F., FRANTZ, U. G.; RODRIGUES, F. A.; FERRER, P. S. Efetividade de um coxim de cabina do trator agrícola na atenuação das vibrações. Revista Ciência Agronômica, v. 45, n. 3, p. 461-468, 2014b.

RIBAS, R. L.; SCHLOSSER, J. F.; FRANTZ, U. G.; FARIAS, M. S. Exposição humana às vibrações de corpo inteiro em um trator agrícola com pneus radiais. Ciência Rural, Santa Maria-RS, v. 44, n. 9, p.1589-1595, 2014.

SANTOS, L. N.; FERNANDES, H. C.; SOUZA, A. P.; FURTADO JÚNIOR, M. R.; SILVA, R. M. F. Avaliação dos níveis de ruído e vibração de um conjunto trator-pulverizador, em função da velocidade de trabalho. Engenharia na Agricultura. Viçosa-MG. v. 22, n. 2, p. 306-313. 2014.

SANTOS FILHO, P. F. FERNANDES, H. C.; QUEIROZ, D. M.; SOUZA, A. P.; CAMILO, A. J. Avaliação dos níveis de vibração vertical no assento de um trator agrícola de pneus utilizando um sistema de aquisição automática de dados. Revista Árvore, Viçosa-MG, v. 27, n. 6, p. 887-895, 2003.

SCARLETT, A. J., PRICE, J. S., STAYNER, R. M. Wholebody vibration: Evaluation of emission and exposure levels arising from agricultural tractors. Journal of Terramechanics. Silsoe-UK, v. 44, p. 65-73, 2007.

SILVA, C. B. VOLPATO, C. E. S.; ANDRADE, L. A. B.; BARBOSA, J. A. Avaliação ergonômica de uma colhedora de cana-de-açúcar. Ciência e Agrotecnologia, Lavras-MG, v. 35, n. 1, p 179-185, 2011.

TEWARI, V. K.; DEWANGAN, K. N. Effect of vibration isolators in reduction of work stress during field operation of hand tractor. Biosystems Engineering, v. 103, p. 146-158, 2009.

ZEHSAZ, M.; SADEGHI, M. H.; ETTEFAGH, M. M.; SHAMS, F. Tractor cabin's passive suspension parameters optimization via experimental and numerical methods. Journal of Terramechanics, Amsterdã, v. 48, p. 439-450, 2011. 